\title{
Factores ambientales condicionantes del metabolismo de una laguna pampeana
}

\author{
María L. Padulles ${ }^{12,}{ }^{\varpi}$; Ana Torremorell ${ }^{12,}$; Virginia Pozzobón ${ }^{1}$; Belena Díaz ${ }^{3}$ \\ \& Adonis Giorgi ${ }^{1 / 2}$ \\ ${ }^{1}$ Instituto de Ecología y Desarrollo Sustentable (INEDES). CONICET-Universidad Nacional de Luján. ${ }^{2}$ Departamento de \\ Ciencias Básicas, Universidad Nacional de Luján. ${ }^{3}$ ANPCyT. Facultad de Ciencias Exactas y Naturales (UBA).
}

\begin{abstract}
RESUMEN. Las concentraciones naturales de fósforo y nitrógeno en las lagunas pampeanas permiten el desarrollo de densas praderas de vegetación acuática sumergida, el dominio de fitoplancton o plantas acuáticas flotantes. En las últimas décadas, la eutrofización de las lagunas aumentó con el avance de labores agrícolas, fertilización de campos y el stock ganadero. La turbidez que producen las floraciones fitoplanctónicas inhibe el crecimiento de macrófitas sumergidas y el fitoplancton domina. Este es el caso de la laguna de Lobos, que por más de 20 años se mantuvo como una laguna turbia orgánica con predominio de cianobacterias. Estudiar el metabolismo ecosistémico aporta gran información sobre el modo en que el estado trófico de la laguna contribuye a la producción, respiración y metabolismo neto, con posibles consecuencias para la biota acuática. Aquí presentamos una evaluación del metabolismo en esta laguna a partir de los cambios en la concentración de oxígeno disuelto, estimado para otoño y primavera. La producción bruta de la laguna fue menor y menos variable en otoño $\left(1.57 \pm 1.33 \mathrm{~g} \mathrm{O}_{2} \cdot \mathrm{m}^{-2}\right.$. día $\left.\mathrm{a}^{-1}\right)$ que en primavera $\left(20.45 \pm 18.00 \mathrm{~g} \mathrm{O}_{2} \cdot \mathrm{m}^{-2}\right.$.día $\left.{ }^{-1}\right)$. Si bien la producción fue alta durante el día, la respiración también fue elevada - incluso, la superó- sobre todo en primavera, cuando fue mayor la densidad de organismos planctónicos, principalmente cianobacterias. Por ello, el metabolismo neto tuvo valores bajos o negativos. Esta situación de gran consumo de oxígeno se acentuó en el período de mayor temperatura y de menor profundidad de la laguna, que coincide con la mayor concentración de clorofila-a y de material particulado, y la menor transparencia del agua. Concluimos que la laguna fue predominantemente heterotrófica durante el período que duró el estudio. De mantenerse las altas concentraciones de nutrientes es probable que aumenten los factores que producen estrés y sus consecuencias negativas para la fauna acuática.
\end{abstract}

[Palabras clave: laguna turbia orgánica, fitoplancton, eutrofización, producción primaria, respiración y oxígeno disuelto]

\begin{abstract}
Environmental factors conditioning the metabolism of a Pampean shallow lake. Pampean shallow lakes naturally have water nitrogen and phosphorous concentrations that allow the development of dense beds of submerged aquatic vegetation, phytoplankton, or free-floating aquatic plants. In recent years, the eutrophication of these shallow lakes has increased due to the intensification of agriculture, use of fertilization and livestock. Phytoplankton- induced turbidity inhibits the growth of the submerged aquatic vegetation leading to phytoplankton dominance. This is the case of the Lobos shallow lake, which in a period greater than 20 years has remained as an organic turbid shallow lake, with cyanobacteria dominance. The study of the ecosystem metabolism can give relevant information about how the trophic state of a shallow lake drives production, respiration and net metabolism, and the potential consequences for aquatic biota. We evaluated the Lobos shallow lake metabolism using diel changes in dissolved oxygen concentrations during autumn and spring. Gross primary production showed lower values and variability in Autumn $\left(1.57 \pm 1.33 \mathrm{~g} \mathrm{O}_{2} \cdot \mathrm{m}^{-2}\right.$.day $\left.{ }^{-1}\right)$ than in Spring $\left(20.45 \pm 18.00 \mathrm{~g} \mathrm{O}_{2} \cdot \mathrm{m}^{-2}\right.$. day $\left.{ }^{-1}\right)$. Although there was high production during the day, respiration was also high, especially in Spring, where there was higher density of planktonic organisms principally cyanobacteria, exceeding net production in both seasons. Thus, we found mainly low or negative values for the net metabolism in Lobos shallow lake. A high oxygen consumption occurred during spring and at the lowest depth, coinciding with the highest chlorophyll-a concentrations, particulate matter, and lowest water transparency. We conclude that Lobos shallow lake was predominantly heterotrophic during study period. High nutrient concentrations will likely increase the factors that produce stress and negative impacts on aquatic fauna.
\end{abstract}

[Keywords: turbid organic lake, phytoplankton, eutrophication, primary production, respiration and dissolved oxygen] 


\section{INTRODUCCIÓN}

El estudio del metabolismo en los sistemas acuáticos aportainformación sobrelos procesos ecológicos, la integridad de los ecosistemas, el grado de deterioro y sus posibilidades de recuperación (Ahearn et al. 2006; Staher et al. 2010). A nivel ecosistémico, en los ambientes acuáticos, las variaciones diurnas de la concentración de oxígeno disuelto (OD) o metabolismo diurno reflejan el balance biológico entre producción y respiración, así como el intercambio físico del oxígeno entre el aire y el agua. Dicho metabolismo varía en la escala diaria y estacional (Cole et al. 2000). Si bien las diferencias en metabolismo entre ecosistemas, así como los cambios a escala anual o estacional, se estudiaron durante varias décadas, hay menos información sobre los cambios en el corto plazo, fundamentalmente a escala diurna. Además, es necesario profundizar en saber de qué modo las diferencias diarias en la irradiancia, la temperatura y los vientos afectan tanto a la producción como a la respiración en un ambiente eutrófico.

Se espera que las lagunas presenten variaciones importantes de oxígeno y dióxido de carbono debido a su pequeño volumen de agua y al contacto estrecho con el sedimento (Pace and Praire 2005). En estos ambientes, el fitoplancton, las macrófitas o el perifiton controlan la producción bruta, y la materia orgánica puede influir mucho sobre la respiración (Coloso et al. 2011). En lagunas con alto contenido de nutrientes se registraron elevados niveles de producción y respiración (Staher et al. 2012). Sin embargo, en muchas lagunas, el metabolismo resulta netamente heterotrófico, con valores de producción neta menores que los de respiración (Hagerthey et al. 2010; Scharfenberger et al. 2019). Por lo general, a las lagunas turbias (i.e., en las que la luz solar no llega hasta el fondo) se las ha asociado a un metabolismo heterotrófico (Herrera and Nadaoka 2021). En la Región Pampeana argentina también hay lagunas turbias. En una de ellas se estudió el metabolismo y se encontró que la respiración es mayor que la producción neta (Alfonso et al. 2018).

En la Región Pampeana existen tres tipos de lagunas: las de aguas claras, las de aguas turbias inorgánicas y las de aguas turbias orgánicas o dominadas por fitoplancton (Allende et al. 2009); esta última categoría reúne a la mayor parte de las lagunas pampeanas. Si bien estas lagunas naturalmente tienen un elevado contenido de nutrientes, décadas atrás se podían encontrar sistemas dominados por macrófitas o fitoplancton producto de una competencia entre ambas comunidades (Izaguirre and Vinocur 1994a). Sin embargo, en los últimos 70 años, la mayoría de estos ecosistemas sufrieron procesos acelerados de eutrofización como consecuencia del incremento en las descargas de aguas provenientes de actividades agrícolas, urbanas e industriales. Al igual que en otras partes del mundo, el incremento de nutrientes en la mayoría de las lagunas pampeanas provocó la exclusión de macrófitas como consecuencia del aumento de la biomasa del fitoplancton y del material suspendido (Quirós et al. 2002; González Sagrario et al. 2020).

La laguna de Lobos es un caso representativo de los procesos que han sufrido las lagunas pampeanas, que se han estacionado en una fase de 'aguas turbias orgánicas'. La reducción de la profundidad de la laguna por causa de factores naturales o antrópicos (e.g., condiciones de sequía o la regulación del caudal de ingreso y salida) produce un ambiente propicio para el desarrollo de cianobacterias. Como se describió para otras lagunas pampeanas, el predominio de este tipo de comunidades impide la colonización de macrófitas sumergidas que podrían modificar la turbidez del agua, el régimen de temperaturas y el estado de equilibrio de la laguna, llevándolo a un estado permanente de laguna turbia orgánica (Diovisalvi et al. 2010; Torremorell et al. 2009; O'Farrell et al. 2011).

El estado trófico de la laguna de Lobos condiciona su funcionamiento. Debido a las altas concentraciones de nutrientes es esperable un alto grado de captación de carbono y de liberación de oxígeno durante las horas de luz por parte de la gran biomasa de fitoplancton, la que genera una alta producción fotosintética (como se encontró en lagunas pampeanas de condiciones similares) (Allende et al. 2009; Torremorell et al. 2018). Además, la región posee un clima templado que posibilita condiciones de alto consumo de oxígeno durante el día y durante la noche como consecuencia de la respiración del plancton y de organismos bentónicos (Zbikowski et al. 2019). También se espera que la producción y el consumo sean más altos en los períodos de mayor irradiancia y temperatura. La mayor irradiancia permite recibir más luz y a su vez incrementa la temperatura, que tiene efectos tanto en la producción bruta como en 
la respiración (Carignan et al. 2000). Nuestro objetivo fue evaluar los cambios diarios en el metabolismo de la laguna en dos períodos del año con diferentes temperaturas, como una primera aproximación para interpretar el funcionamiento de la misma y explorar qué factores se asocian a dicho funcionamiento.

En base a lo mencionado se plantean las siguientes hipótesis: 1) una mayor biomasa de fitoplancton genera una diferencia más pronunciada de la relación producción primaria/respiración entre el día y la noche, con posible saturación en el día y posible anoxia en la noche; 2) el metabolismo neto diario de la laguna es heterotrófico, ya que al ser una laguna turbia orgánica tendrá una gran cantidad de material en suspensión y de organismos plantónicos que consumen oxígeno durante el día y la noche, ausencia de vegetación en el fondo y escasa penetración de la luz, que limita la producción en el día.

\section{Metodología}

\section{Área de estudio}

La laguna de Lobos se ubica en la Región Pampeana, en el área denominada Pampa Deprimida, al noroeste de la provincia de Buenos Aires, y pertenece a la cuenca del Río
Salado (Frenguelli 1950). Es un centro turístico donde actualmente se realizan actividades recreativas, turísticas y deportivas importantes para la localidad y la región (Pozzobon and Cabrera 2013). Su volumen de agua $\left(8.6 \mathrm{hm}^{3}\right)$ depende de distintos procesos hidrológicos como el ingreso de su principal afluente, el arroyo Las Garzas (Figura 1), al norte de la laguna, y del arroyo Salvador María, al oeste, de las precipitaciones y de la evaporación (Pozzobon 2009). La superficie estimada es $7.5 \mathrm{~km}^{2}$, su profundidad media es $\sim 1 \mathrm{~m} \mathrm{y}$ su profundidad máxima es $1.46 \mathrm{~m}$. Por esta razón se considera que la laguna de Lobos es un cuerpo de agua léntico, polimíctico y eutrófico de la zona pampeana, con ausencia de estratificación y con importantes variaciones térmicas diarias, mientras que por su salinidad se la caracteriza como oligohalina y poiquioligolohalina (Boltovskoy et al. 1990; Mariñelarena and Conzonno 1997).

Actualmente, y desde hace 25 años, la laguna viene sufriendo un acelerado proceso de eutrofización, hecho plasmado por diferentes autores (Boltovskoy et al. 1990; Izaguirre and Vinocur 1994a; Izaguirre and Vinocur 1994b; Pozzobon 2009; Pozzobon and Cabrera 2013). La caracterización realizada por Pozzobon (2009) se corresponde con un cuerpo de agua eutrófico, de $\mathrm{pH}$ alcalino, con

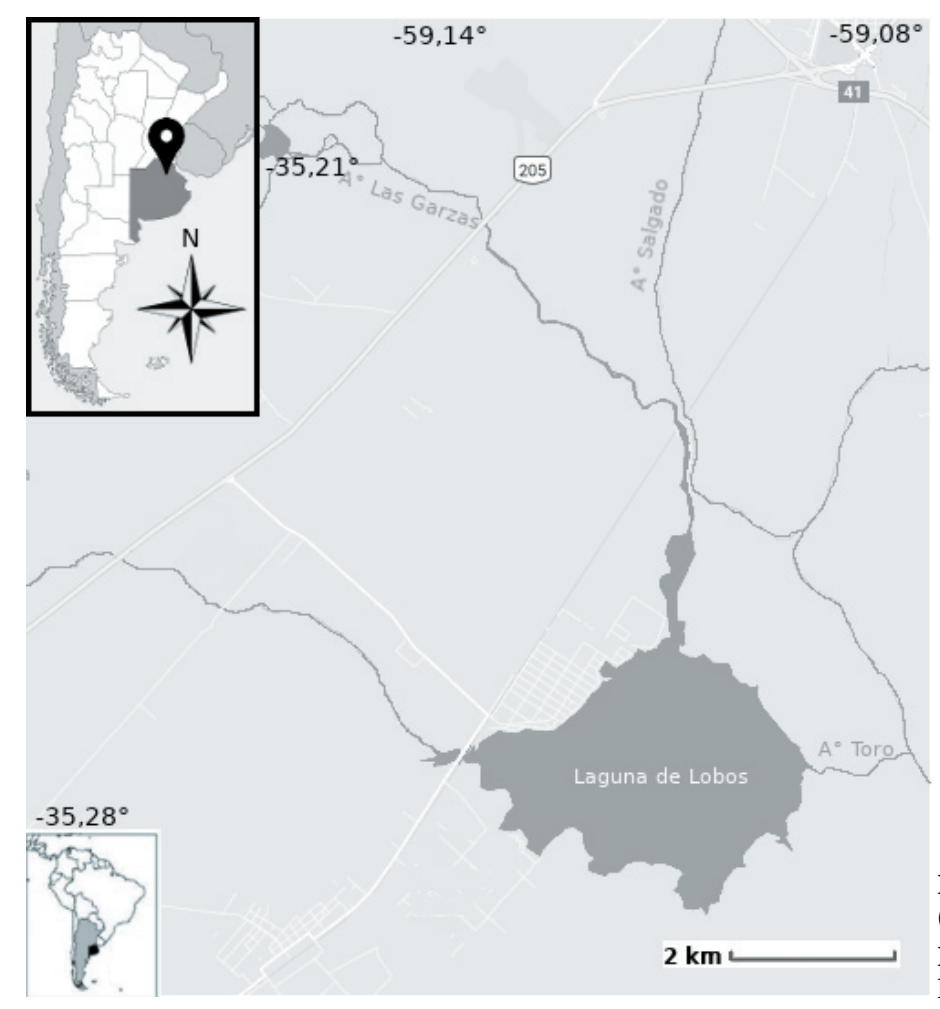

Figura 1. Localización del sitio de estudio (laguna de Lobos).

Figure 1. Study site location (Lobos shallow lake). 
predominio de cianobacterias filamentosas. En el mismo trabajo se señala la influencia que ejerce el arroyo Las Garzas en los parámetros físicos, químicos y fitoplanctónicos por su bajo contenido de oxígeno y organismos provenientes de efluentes cloacales. Este aspecto también fue destacado por Mariñelarena (1990) y por Mariñelarena y Conzonno (1997). Cabe destacar que en este último estudio se cita un valor promedio de clorofila planctónica de $155.77 \mu \mathrm{g} / \mathrm{L}$, así como altas concentraciones de fosfatos $(30 \mu \mathrm{g} / \mathrm{L})$ y de amonio $(390 \mu \mathrm{g} / \mathrm{L})$, por lo que sus autores lo consideran un sistema altamente eutrófico o hipereutrófico.

\section{Diseño de muestreo}

Se realizó un seguimiento del estado de la laguna en dos épocas del año: otoño y primavera (del 23 de abril al 3 de mayo de 2016 y del 13 al 23 de diciembre de 2016, respectivamente). En un sitio central de la laguna, siempre al mediodía y durante 10 días seguidos, se registraron parámetros físicos como la temperatura, la conductividad y el $\mathrm{pH}$ utilizando una sonda multiparamétrica Thermo Orion, y se estimó la transparencia del agua mediante disco de Secchi.

De igual modo, cada día se tomaron muestras de agua para determinar las concentraciones de fósforo reactivo soluble (método del ácido ascórbico) y de amonio (método del fenol) (APHA 1995). También se estimaron diariamente la biomasa del seston y su porcentaje de contenido orgánico e inorgánico (MPS orgánico e inorgánico) mediante filtrado de agua con filtros de fibra de vidrio GF/C secados y pre-pesados. Además, se estimó la biomasa fitoplanctónica por espectrofotometría luego de la extracción de la clorofila-a con acetona al $90 \%$ a una muestra de agua filtrada mediante filtros de fibra de vidrio GF/F. La determinación correspondió a la clorofila activa, ya que se acidificó la muestra para descontar los feopigmentos (APHA 1995).

\section{Velocidad del viento}

Todos los días se midió la velocidad del viento con un anemómetro manual marca Luthrom LM 8000 a una altura de 2 m, de acuerdo con la dirección predominante de los vientos en cada oportunidad de medición. La velocidad del viento a $10 \mathrm{~m}$ desde la base fue estimada por la ley de potencia de perfil de viento utilizada por Cole et al. (2010).

\section{Curva diaria de oxígeno y metabolismo del ecosistema}

El OD y la temperatura se midieron usando una sonda Hach HQ40 multiparamétrica previamente calibrada. La sonda se colocó durante los 10 días de muestreo en el centro de la laguna a $0.5 \mathrm{~m}$ de profundidad para tomar medidas de modo automático cada 5 minutos durante períodos de 24 horas. Finalizado este período se cambiaba el equipo registrador por otro de iguales características para continuar utilizando la misma sonda. A partir de los datos de OD, temperatura, porcentaje de saturación y presión atmosférica se pudo estimar el metabolismo neto del ecosistema $(\mathrm{MN})$, la respiración del ecosistema (RE) y su producción bruta (PB).

Para estimar el metabolismo se tuvo en cuenta la variación de oxígeno en el agua cada 5 minutos y su relación con el valor teórico de saturación de oxígeno en función de la temperatura del agua y la altura sobre el nivel del mar. Cuando el valor medido de oxígeno disuelto es mayor al valor estimado de saturación, se asume una condición de sobresaturación y de emisión del gas a la atmósfera. En cambio, si es menor, se espera que ingrese oxígeno desde la atmósfera al cuerpo de agua. Este valor de intercambio mediante la concentración de oxígeno presente en el agua y la atmósfera se estima mediante el coeficiente de reaereación que se explica más abajo y que varía con la velocidad del viento. La sumatoria de las variaciones de oxígeno multiplicada por el coeficiente de reaereación indica el flujo del oxígeno en la laguna (D). De ese modo se puede aplicar la ecuación original de Odum (1956) (ver Hoellein et al. 2013).

$$
\delta \mathrm{O}_{2} / \delta \mathrm{t}=\mathrm{PB}-\mathrm{RE}-\mathrm{D}
$$

donde $\delta \mathrm{O}_{2} / \delta$ t es la variación de oxígeno en el tiempo, que está dada por PB (la producción bruta), por RE (la respiración del ecosistema) y por D (el flujo de oxígeno) (Staher et al. 2010).

El valor obtenido por la ecuación corresponde a la variación de la concentración de oxígeno $\left(\mathrm{g} / \mathrm{m}^{3}\right)$ en el intervalo de 5 minutos. Para determinar la respiración se asumió que de noche no hay fotosíntesis, sino únicamente respiración, y que no hay diferencias en la respiración entre el día y la noche, entendiendo el período de la noche como las horas posteriores a la caída del sol y previas a su salida. Se promediaron los valores de oxígeno del horario nocturno y en base a eso 
se estimó el valor de respiración por hora. La RE se calculó por la sumatoria de la respiración por hora durante las $24 \mathrm{~h}$. La PB se calculó como la suma de las variaciones de oxígeno durante las horas de luz, corregidos por los coeficientes de intercambio y sumadas a los valores de respiración de las 24 horas del día. La PB es atribuible a la producción del fitoplancton o de la vegetación sumergida en la laguna, en caso de que hubiera. Su valor puede ser 0 , pero nunca negativo. Por otro lado, se calculó la diferencia entre PB y RE para obtener el MN, que puede ser positivo (cuando la producción es mayor a la respiración), negativo (cuando es mayor el consumo que la producción) ó 0 cuando la producción y la respiración están igualadas (Coloso et al. 2008; Hoellein et al. 2013).

Los valores de PB y RE en cada intervalo de 5 minutos deben corregirse por el valor del flujo de oxígeno o difusión (D). Para estimarlo se utilizaron las ecuaciones planteadas en Cole et al. (2010) y Staehr et al. (2010). Así, Dt(0.12 h) sería la difusión de $\mathrm{O}_{2}$ entre el agua de la laguna y la atmósfera en períodos de 0.12 horas (5 minutos), y se calculó de acuerdo a la ecuación que brindan Van de Bogert et al. (2007).

$$
\operatorname{Dt}(0.12 \mathrm{~h})=\mathrm{k}\left(\mathrm{O}_{2} \text { sat }-\mathrm{O}_{2} \mathrm{t}\right) \cdot \mathrm{z}^{-1}
$$

donde $\mathrm{k}$ es el coeficiente de intercambio de gas $\mathrm{O}_{2}$ a una temperatura determinada (Cole and Caraco 1998), O sat es la concentración de oxígeno de equilibrio del agua para esa condición y en ese momento, $\mathrm{O}_{2}$ t es la medida de concentración de oxígeno en un momento dado (t) y z es la profundidad media de la laguna. El parámetro k se calculó para cada temperatura del agua utilizando la ecuación de Klotz (2013).

$$
\mathrm{k}=\mathrm{k}_{600}[\mathrm{Sc} / 600]^{-1 / 2}
$$

donde $\mathrm{k}_{600}$ es el coeficiente de intercambio de oxígeno para un número de Schmid de 600 y Sc es el número de Schmidt para oxígeno a diferentes temperaturas del agua, que se determina por medio de la ecuación de Wanninkhof (1992).

$$
\mathrm{Sc}_{\mathrm{C}}=0.0476 \mathrm{~T} 3+3.7818 \mathrm{~T} 2-120.1 \mathrm{~T}+1800.6
$$

donde $\mathrm{T}$ es la temperatura en grados centígrados.

Los valores de $\mathrm{k}_{600}$ se calcularon utilizando la ecuación de Cole and Caraco (1998). $\mathrm{k}_{600}=\left(2.07+0.215 \mathrm{U} 10^{1.7}\right) \cdot 100^{-1}$

donde U10 es la velocidad del viento a $10 \mathrm{~m}$ de altura. Es decir que RE se obtiene a partir de la variación nocturna de oxígeno, asumiendo que se mantiene durante todo el día, PB se obtiene de la producción durante las horas de luz y la respiración durante 24 horas, y MN se obtiene por diferencia entre ambas. Para realizar estas estimaciones siempre debe corregirse por D (el oxígeno que es intercambiado con la atmósfera) y considerar el valor z (la profundidad de la laguna) para obtener una estimación del metabolismo de la laguna. Las estimaciones de metabolismo realizadas se expresan en $\mathrm{g} \mathrm{O}_{2} \mathrm{~m}^{-2}$.día ${ }^{-1}$. Esa unidad se obtiene al multiplicar los resultados de concentración de oxígeno estimados $\left(\mathrm{g} / \mathrm{m}^{3}\right)$ por la profundidad de mezcla de la laguna (en $\mathrm{m}$ ), obteniéndose la variación de oxígeno por unidad de área estimada para toda la laguna. De ese modo pueden compararse los resultados del metabolismo entre lagunas de distintas superficies y profundidades.

\section{Análisis estadísticos}

Respecto a la variación diaria del OD, se registraron los valores de concentración promedio y su desvío estándar en el transcurso del día, así como los valores máximos y mínimos de concentración de oxígeno y su coeficiente de variación. También se contabilizó si hubo días con sobresaturación o agotamiento de oxígeno o con valores menores a $5 \mathrm{mg} / \mathrm{L}$ de OD, nivel guía para preservar la biota acuática.

Las relaciones entrelas variables seexploraron mediante un análisis de componentes principales (ACP). Para evitar redundancia, en el análisis no se incorporaron las variables temperatura, material particulado orgánico y material particulado inorgánico. Por otro lado, se utilizó el logaritmo decimal para normalizar las variables amonio, profundidad y conductividad. También se realizaron comparaciones entre los parámetros de metabolismo de otoño y primavera mediante análisis de la varianza. Tanto para ellas como para las comparaciones de variación de oxígeno durante el día se utilizó el programa InfoSTAT 2020 y para las representaciones gráficas el programa Sigma Plot 8.0.

\section{Resultados}

Durante el estudio se observó que la laguna frecuentemente se sobresatura de oxígeno 
en algún momento del día. En otoño, la concentración de oxígeno no disminuye como para comprometer la vida de los peces; sin embargo, en primavera, seis de los diez días que se monitorearon presentaron concentraciones de oxígeno menores a $3 \mathrm{mg} / \mathrm{L}$. En ningún momento la laguna llegó a estar totalmente anóxica. Tanto los valores de oxígeno a lo largo del día como su amplitud y su coeficiente de variación fueron significativamente menores en otoño que en primavera. En la Tabla 1 se sintetiza información relacionada con el OD en la laguna, extraída de las curvas diarias de oxígeno de ambos períodos.

El MN promedio durante los diez días de otoño fue -0.75 (d.e. 1.72) $\mathrm{g} \mathrm{O}_{2} \cdot \mathrm{m}^{-2}$.día ${ }^{-1}$, mientras que en primavera fue -1.14 (d.e. 11.72) $\mathrm{gO}_{2} \cdot \mathrm{m}^{-2} \cdot$ día $^{-1}$. Los máximos valores diarios de producción bruta calculados en otoño y primavera fueron 4.51 y $59.93 \mathrm{~g} \mathrm{O}_{2} \cdot \mathrm{m}^{-}$ ${ }^{2}$. día ${ }^{-1}$, respectivamente, y los mínimos fueron 0.07 y $6.11 \mathrm{~g} \mathrm{O}_{2} \cdot \mathrm{m}^{-2}$.día ${ }^{-1}$, respectivamente. El metabolismo en la laguna resultó negativo en 13 de los 20 días que se estudió, mientras que en los otros 7 días fue positivo (Figura 2).

La mayoría de los días registrados en otoño en laguna tuvieron mayor RE que PB $(\mathrm{PB} / \mathrm{RE}<1)$, mientras que en primavera, la $\mathrm{PB}$ fue mayor que la RE $(\mathrm{PB} / \mathrm{RE}<1)$ (Figura $3)$. En general, las variables consideradas presentaron diferencias significativas entre las distintas estaciones. Sin embargo, no ocurrió así con el OD, que en ambas estaciones presentó valores de saturación, ni tampoco con el fósforo reactivo soluble, que se mantuvo con valores elevados en ambos períodos. $\mathrm{El} \mathrm{pH}$, la temperatura, la velocidad del viento, la conductividad, la clorofila-a y el amonio fueron mayores en primavera. La profundidad de la laguna en primavera fue un tercio menor que en otoño y la profundidad del disco de Secchi disminuyó a la mitad. El material particulado en suspensión total, orgánico e inorgánico fue mayor en primavera
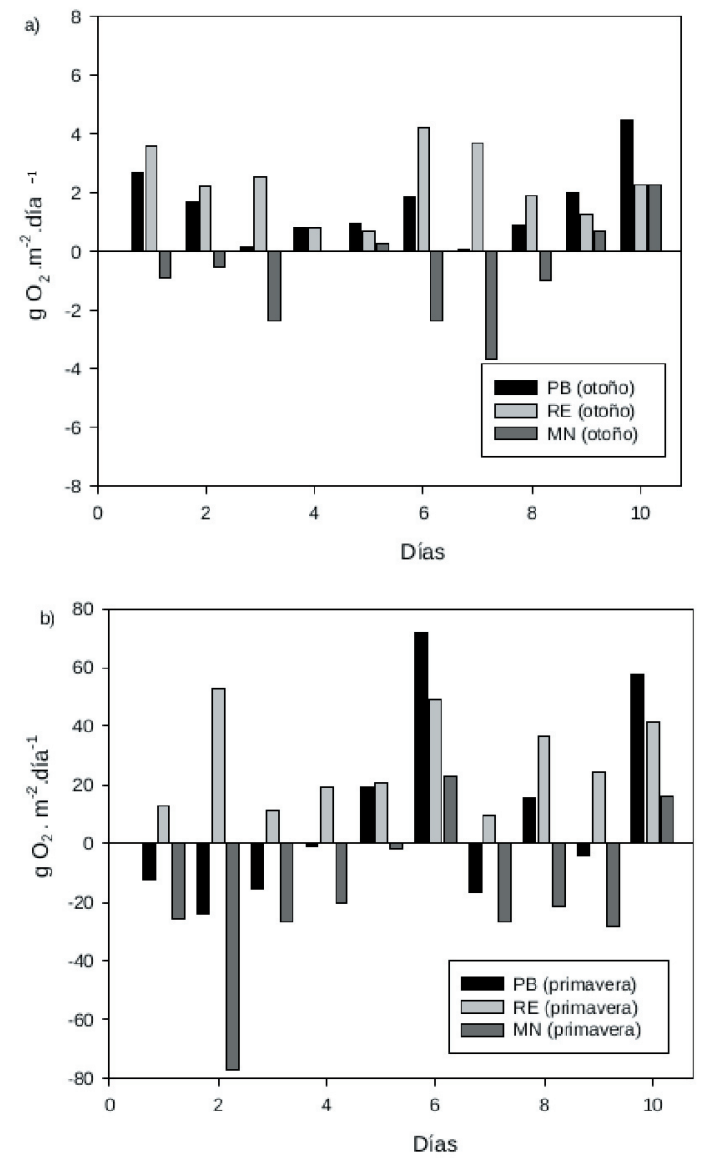

Figura 2. Metabolismo neto (MN), producción bruta $(\mathrm{PB})$ y respiración (RE) durante los 10 días de medición en a) otoño y b) primavera.

Figure 2. Net metabolism (MN), gross production $(\mathrm{PB})$ and respiration (RE) during the 10 days of measurement in a) Autumn and b) Spring.

(Tabla 2). También se obtuvieron valores de PB y RE más altos en primavera, mientras que el metabolismo neto fue menor en primavera en relación al estimado para otoño (Tabla 2 y Figura 2).

El ACP permitió obtener dos ejes en los que las variables se ubicaron principalmente

Tabla 1. Características de las curvas de oxígeno registradas diariamente en períodos de otoño y primavera de 2016. Para la variación de oxígeno disuelto (OD) se indica el valor medio junto al valor del desvío estándar.

Table 1. Characteristics of the oxygen curves recorded daily in the periods of Autumn and Spring of 2016. For dissolved oxygen variation, the mean value and standard deviation value are indicated.

\begin{tabular}{lcc}
\hline & Otoño & Primavera \\
\hline Días con sobresaturación & 9 & 10 \\
Días con OD menor a $3 \mathrm{mg} \mathrm{O}_{2} / \mathrm{L}$ & 0 & 6 \\
Horas diarias sin OD & 0 & 0 \\
Variación diaria de OD $\left(\mathrm{mg} \mathrm{O}_{2} / \mathrm{L}\right)$ & $3.6 \pm 2.2$ & $15.6 \pm 3.1^{*}$ \\
Amplitud diaria de la variación de OD $\left(\mathrm{mg} \mathrm{O}_{2} / \mathrm{L}\right)$ & $0.95-7.95$ & $9.07-20.92^{*}$ \\
Coeficiente de variación de oxígeno $(\%)$ & $3.04-18.17$ & $24.81-60.29^{*}$ \\
\hline
\end{tabular}


Tabla 2. Valores promedio y desvío estándar de las variables físicas, químicas, biológicas y ecológicas registradas en ambos períodos de muestreo, y resultado del análisis de varianza. ns: sin diferencias significativas; ${ }^{*} \mathrm{P}<0.05 ;{ }^{* *}: \mathrm{P}<0.05$; \pm : desvío estándar correspondiente a cada valor medio.

Table 2. Average values and standard deviation of the registered physical, chemical, biological and ecological variables recorded in both periods, and variance analysis. ns: no significant differences; ${ }^{*}$ : significant differences $(\mathrm{P}<0.05)$; ${ }^{* *}$ : highly significant differences $(\mathrm{P}<0.05)$; \pm : standard deviation value corresponding to each mean value.

\begin{tabular}{|c|c|c|c|}
\hline & Otoño & Primavera & \\
\hline $\mathrm{pH}$ & $8.8 \pm 2$ & $10.01 \pm 0.6$ & ** \\
\hline Temperatura $\left({ }^{\circ} \mathrm{C}\right)$ & $14 \pm 1.4$ & $25 \pm 1.8$ & ** \\
\hline Conductividad (mS/cm) & $1.59 \pm 0.14$ & $3.87 \pm 0.1$ & $* *$ \\
\hline Oxígeno disuelto (mg/L) & $11.3 \pm 1.1$ & $11.6 \pm 3.73$ & ns \\
\hline Profundidad (m) & $1.8 \pm 0.08$ & $1.24 \pm 0.01$ & ** \\
\hline Secchi $(\mathrm{cm})$ & $19 \pm 5.3$ & $8.5 \pm 1.5$ & $* *$ \\
\hline Velocidad del viento $(\mathrm{km} / \mathrm{s})$ & $3.3 \pm 1.8$ & $8.9 \pm 5.5$ & * \\
\hline Dirección dominante del viento & $\mathrm{Sy} \mathrm{SO}$ & No la hay & \\
\hline Material particulado en suspensión (mg/L) & $0.53 \pm 0.26$ & $13.49 \pm 3.44$ & $* *$ \\
\hline Material inorgánico en suspensión (mg/L) & $0.31 \pm 0.19$ & $9.2 \pm 1.80$ & $* *$ \\
\hline Material orgánico en suspensión (mg/L) & $0.21 \pm 0.1$ & $4.29 \pm 1.87$ & $* *$ \\
\hline Clorofila-a $(\mu \mathrm{g} / \mathrm{L})$ & $290 \pm 110.1$ & $762.3 \pm 158.5$ & ** \\
\hline Fósforo reactivo soluble (mg/L) & $0.15 \pm 0.079$ & $0.18 \pm 0.016$ & ns \\
\hline Amonio $(\mu \mathrm{g} / \mathrm{L})$ & $2 \pm 1.4$ & $217.2 \pm 190.4$ & * \\
\hline Producción bruta $\left(\mathrm{g} \mathrm{O}_{2} \cdot \mathrm{m}^{-2} \cdot\right.$ día $\left.^{-1}\right)$ & $1.57 \pm 1.33$ & $20.45 \pm 18.00$ & $* *$ \\
\hline Respiración del ecosistema $\left(\mathrm{g} \mathrm{O}_{2} \cdot \mathrm{m}^{-2} \cdot\right.$ día $\left.^{-1}\right)$ & $2.32 \pm 1.22$ & $21.59 \pm 14.22$ & $* *$ \\
\hline Metabolismo neto $\left(\mathrm{g} \mathrm{O}_{2} \cdot \mathrm{m}^{-2} \cdot\right.$ día $\left.^{-1}\right)$ & $-0.8 \pm 1.72$ & $-1.14 \pm 11.62$ & ns \\
\hline
\end{tabular}

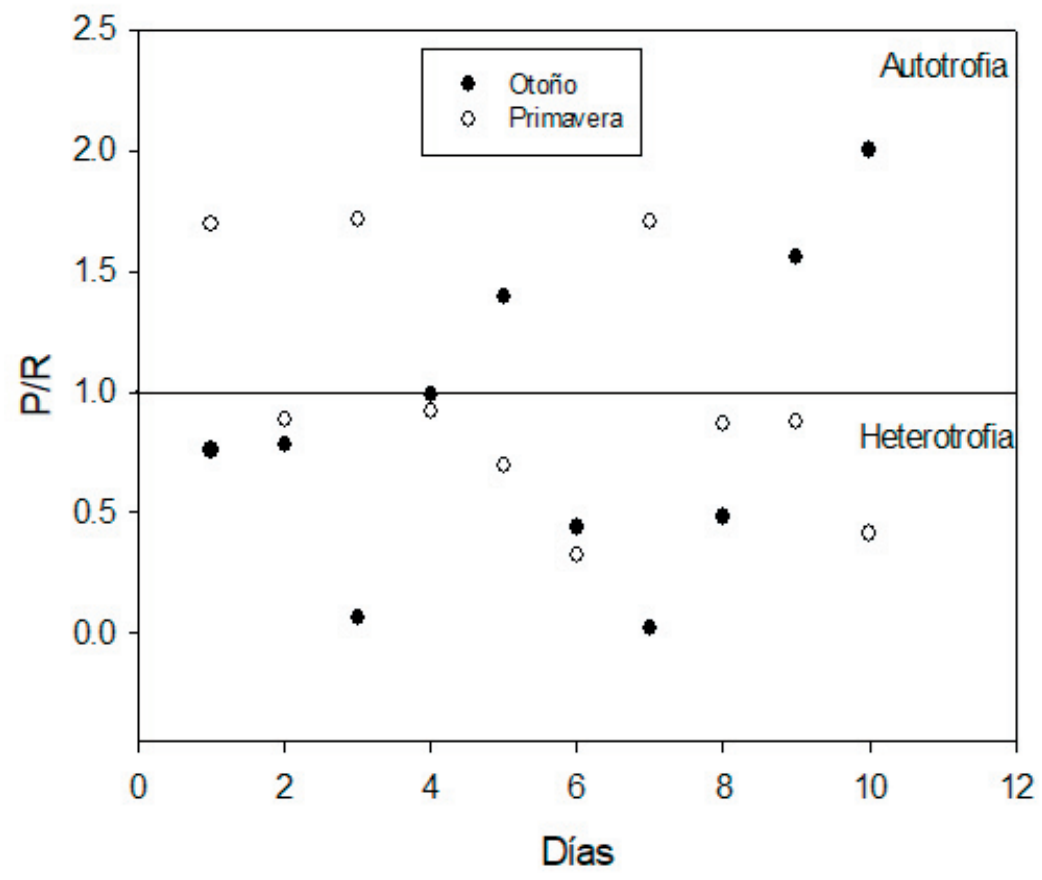

Figura 3. Representación del índice de producción bruta y respiración $(\mathrm{P} / \mathrm{R})$ resultante en cada día de otoño y primavera. Los símbolos negros indican los valores del índice en otoño y los claros indican los valores de primavera.

Figure 3. Representation of the gross primary production and respiration index $(\mathrm{P} / \mathrm{R})$ in each day of Autumn and Spring. Black symbols indicate index values at fall and light symbols indicate spring values. 


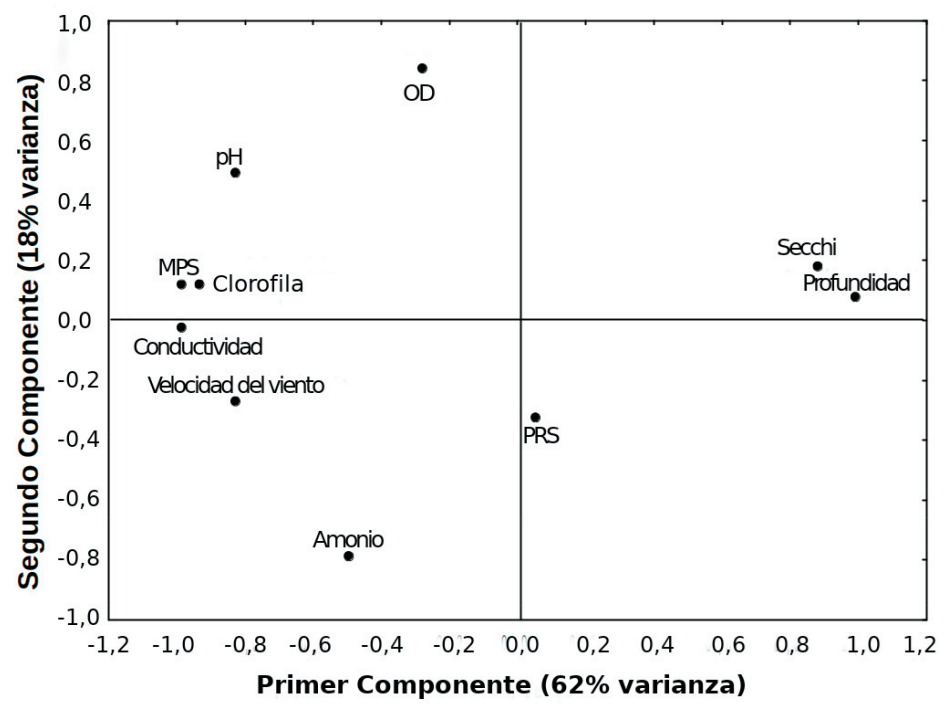

Figura 4. Análisis de componentes principales indicando la distribución de las variables consideradas (MPS: material particulado total; PRS: fósforo reactivo soluble; OD: concentración de oxígeno disuelto). A las variables conductividad, profundidad y amonio se les aplicó logaritmo para normalizarlas.

Figure 4. Principal component analysis ordination of variables considered (MPS: total particulate material; SRP: soluble reactive phosphorus; $\mathrm{OD}$ : concentration of dissolved oxygen). A logarithm was applied to the conductivity, depth and

Primer Componente (62\% varianza)
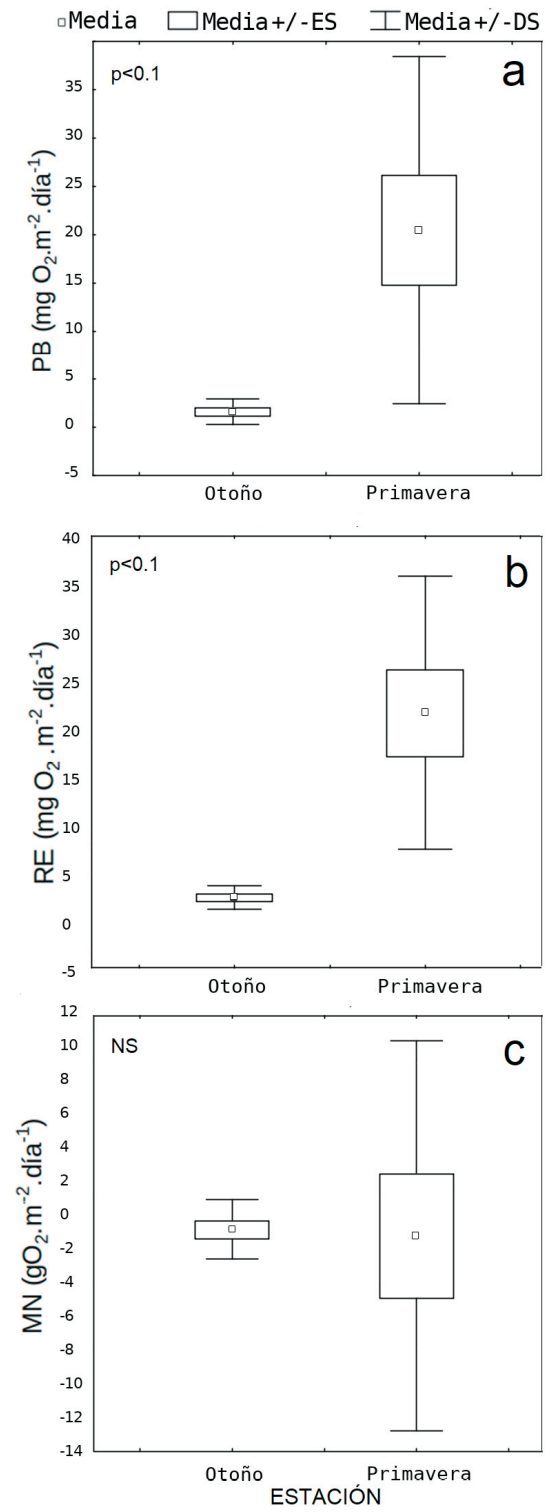

relacionadas al primero, que explicó el $62 \%$ de la varianza, mientras que el segundo eje explicó el $18 \%$, reuniendo entre ambos el $80 \%$ de la varianza. El primer eje se relacionó con el nivel hidrométrico y la turbidez de la laguna. Las variables principales fueron la profundidad de la laguna y la transparencia del disco de Secchi en el cuadrante positivo, mientras que en los cuadrantes negativos fueron el material particulado en suspensión, la conductividad y la clorofila-a. El segundo eje se asoció a la variación diaria y presentó la concentración de amonio y fósforo reactivo soluble (PRS) de modo opuesto a la de OD (Figura 4).

La PB y la RE en primavera resultaron significativamente mayores que en otoño $\mathrm{P}<0.01$. El MN no mostró diferencias significativas entre primavera y otoño. Sin embargo, de igual modo que la PB y la RE, en primavera, su rango de variación diario fue mayor que en otoño (Figura 5).

\section{Discusión}

Utilizando el criterio de Mariñelarena y Conzonno (1997), que tiene en cuenta el pH elevado y las altas concentraciones de oxígeno, clorofila-a y nutrientes, catalogamos a la laguna de Lobos como hipereutrófica dada la presencia de altas concentraciones de fosfatos

Figura 5. Diagrama de cajas de PB (a), RE (b) y MN (c) de otoño y primavera. Los valores de PB y RE son significativamente mayores en primavera $\left(\mathrm{P}^{\prime} 0.01\right)$.

Figure 5. Boxplots of gross primary production (PB) (a), ecosystem respiration (RE) (b) and net metabolism (MN) (c). The PB and RE values are significantly higher in Spring $\left(\mathrm{P}^{\prime} 0.01\right)$. 
y clorofila-a, así como la baja transparencia del disco de Secchi. La variación de OD en la columna de agua da una medida integradora que sintetiza tanto el efecto de factores ambientales como la recirculación producida por los vientos. Si bien en la laguna de Lobos durante nuestro estudio se registraron altas concentraciones de OD durante el día (lo que induciría a suponer que su comportamiento es autotrófico), en ambos períodos, el $\mathrm{MN}$ fue negativo, lo que evidencia una laguna con características heterotróficas debido a la alta tasa de respiración necesaria para procesar la materia orgánica que ingresa a la misma o que se va acumulando con el tiempo. En otras partes del mundo también se encontraron lagunas netamente heterotróficas o que alternan de modo habitual entre autotróficas y heterotróficas (Hagerthey et al. 2010; Laas et al. 2012).

Los cambios del metabolismo diario, tanto en otoño como en primavera, pueden explicarse por la velocidad del viento, que produciría mayor circulación de la masa de agua y variación del material particulado. Según observaciones realizadas en el mismo período de muestreo, el material particulado estuvo conformado por fitoplancton dominado por cianobacterias, pero en períodos de mayor circulación de agua presentó también diatomeas y partículas de sedimentos (Barraza 2019). Los valores de PB, RE y MN se encuentran dentro del rango de variación reportados para otros ecosistemas similares en tamaño y profundidad como, por ejemplo, los hallados por Klotz et al. (2013) para dos lagos someros de Nueva York (EE.UU.), de superficie y profundidad similares a la laguna de Lobos, y por Melack en 2009 para un lago tropical de profundidad comparable, aunque con el doble de superficie. Cabe destacar que en un estudio previo realizado en la laguna La Salada, situada en la provincia de Buenos Aires, con características similares a la de Lobos (i.e., somera, polimíctica, mesotrófica-eutrófica), Alfonso et al. (2015) también encontraron valores extremos de $\mathrm{PB}$ y $\mathrm{RE}$ en otoño (PB: $2.8 \mathrm{~g} \mathrm{O}_{2} \cdot \mathrm{m}^{-2}$. día ${ }^{-1}$, RE: $1.17 \mathrm{~g} \mathrm{O}_{2} \cdot \mathrm{m}^{-2} \cdot$ día $^{-1}$ ) y en primavera (PB: $10.82 \mathrm{~g} \mathrm{O}_{2} \cdot \mathrm{m}^{-2} \cdot$ día $^{-1}$, RE: 5.6 $\mathrm{g} \mathrm{O}_{2} \cdot \mathrm{m}^{-2} \cdot$ día $\left.^{-1}\right)$. Sin embargo, los valores en primavera en laguna de Lobos son más altos. La laguna presenta como uno de los rasgos de eutrofia una gran amplitud en los ciclos diarios de oxígeno que se manifiestan en valores de sobresaturación de oxígeno. Parte de este gas se libera a la atmósfera y se pierde para el sistema acuático, en particular durante los meses más cálidos, cuando hay menor presión parcial de oxígeno. A su vez, la respiración que realizan tanto las comunidades planctónicas como las bentónicas puede consumir gran parte de las reservas de oxígeno en el agua, dado que en esta laguna, algunos de los autores observaron una gran cantidad de peces bentónicos, como la carpa (Cyprinus carpio), así como también depósitos de sedimento blando de al menos $50 \mathrm{~cm}$ de profundidad que podrían sostener una gran biomasa de bacterias. En este sentido, hay que considerar la acción intensa de los microorganismos que actúan en la descomposición de la materia orgánica asociados a sedimentos o a material particulado en suspensión.

El conjunto de factores hallados en el muestreo en el período de temperaturas más altas (primavera), como la baja profundidad del agua, la gran cantidad de sedimentos, las bajas concentraciones de oxígeno y el metabolismo heterotrófico de la laguna podrían explicar parcialmente la importante mortandad de peces observada pocos días después de concluido el estudio.

La relación entre las variables que propone el ACP indicaría un conjunto de variables asociadas al nivel hidrométrico que estructurarían y mantendrían las características de la laguna. De ese modo, cuando el nivel hidrométrico es mayor suele haber mayor transparencia del agua como resultante de la menor concentración de material particulado en suspensión. Por el contrario, con niveles hidrométricos menores, el efecto de la velocidad del viento también es mayor y se incrementa la cantidad de material particulado conformado principalmente por microalgas y cianobacterias, lo que incrementa la concentración de clorofila en el agua. Ambas situaciones repercuten de manera notable en las respuestas funcionales provocando variaciones amplias en la producción y la respiración. La correlación positiva entre PB y RE y el material particulado en suspensión significa que ese material está formado en gran parte por organismos del fitoplancton que incrementan tanto la producción como la respiración. La situación inversa se da cuando se incrementa la profundidad del agua, ya que se reduce el fitoplancton en la columna de agua y su efecto sobre el metabolismo.

La laguna de Lobos posee concentraciones de clorofila-a fitoplanctónica altas, entre 0.3 y $1 \mathrm{mg} / \mathrm{L}$, lo que hace suponer que su comportamiento es autotrófico. Sin embargo, 
en ambos períodos, el metabolismo neto del ecosistema tuvo una mayoría de valores cercanos al equilibrio y varios marcadamente negativos, evidenciando condiciones de heterotrofia en la mayoría de los días estudiados. Si bien sólo se cuenta con dos estudios previos del metabolismo abierto en lagunas de la región (laguna La Salada, que tenía menor concentración de fósforo en agua, vegetación acuática y aguas claras al momento del estudio), se encontró un balance autotrófico positivo durante el año (Alfonso et al. 2015). Sin embargo, en otro período, tanto en La Salada como en la laguna Sauce Grande se registró un metabolismo predominantemente heterotrófico que los autores atribuyen a la carga de nutrientes y de materia orgánica que ingresarían desde las zonas cultivadas (Alfonso et al. 2018). Esta semejanza en el metabolismo entre lagunas puede atribuirse a que son poco profundas y que están sometidas a procesos similares que podrían acelerarse con el incremento de las áreas cultivadas en sus cuencas de drenaje. Particularmente, las lagunas de Lobos y Sauce Grande se pueden considerar turbias orgánicas, que presentan altas concentraciones de fósforo que favorecería el desarrollo de fitoplancton, incrementando tanto la producción como la respiración.

Al igual que muchas lagunas pampeanas, la laguna de Lobos provee numerosos servicios ecosistémicos; en particular, las lagunas son áreas muy importantes de recreación donde la pesca deportiva es una fuente económica y cultural relevante. Sin embargo, estudios previos destacan la situación de eutrofia en que se encuentra la laguna de Lobos desde hace más de 30 años. El resultado obtenido en este estudio indica que si las condiciones heterotróficas se mantienen en el tiempo debido a las altas concentraciones de nutrientes, podrían incrementarse las situaciones de anoxia y su consiguiente perjuicio para las comunidades de organismos acuáticos.

Agradecimientos. El proyecto fue apoyado por la Universidad Nacional de Luján y Agencia Nacional De Promoción de la Investigación, el Desarrollo Tecnológico y la Innovación a través del PICT: 20130775. También contó con la colaboración de integrantes de la Asociación Civil CIALL de la ciudad de Lobos. Se agradecen todas las sugerencias de los revisores y la editora para mejorar la versión original.

\section{ReFERENCIAS}

Ahearn, D. S., J. H. Viers, J. F. Mount, and R. A. Dahlgren. 2006. Priming the productivity pump: flood pulse driven trends in suspended algal biomass distribution across a restored floodplain. Freshwater Biology 51:1417-1433. https: //doi.org/10.1111/j.1365-2427.2006.01580.x.

Alfonso, M. B., A. Vitale, M. Menéndez, V. Perillo, M. Piccolo, and G. Perillo. 2015. Estimation of ecosystem metabolism from diel oxygen technique in a saline shallow lake: La Salada (Argentina). Hydrobiologia 752:223-237. https://doi.org/ 10.1007/s10750-014-2092-1.

Alfonso, M. B., A. Brendel, A. Vitale, C. Seitz, M. Piccolo, and G. Perillo. 2018. Drivers of Ecosystem Metabolism in Two Managed Shallow Lakes with Different Salinity and Trophic Conditions: The Sauce Grande and La Salada Lakes (Argentina). Water 10:1136-1151. https://doi.org/10.3390/w10091136.

Allende, L., G. Tell, H. Zagarese, A. Torremorell, G. Pérez, J. Bustingorry, R. Escaray, and I. Izaguirre. 2009. Phytoplankton and primary production in clear-vegetated, inorganic-turbid, and algal-turbid shallow lakes from the pampa plain (Argentina). Hydrobiologia 624(1):45-60. https://doi.org/10.1007/s10750-008-9665-9.

American Public Health Association (APHA). 1995. Standard Methods for the Examination of Waters and Wastewaters, 19th ed., Washington, D.C.

Barraza, A. 2019. Microalgas y su uso como biondicadoras en la laguna lobos y su microcuenca fluvial (Buenos Aires). Tesis de Especialización en calidad ecológica y restauración de sistemas fluviales. Universidad Nacional de Luján, Buenos Aires, Argentina. Pp. 54.

Boltovskoy, A., A. Dippolito, A. Foggeta, M. Gómez, and N. Álvarez, G. 1990. La laguna Lobos y su afluente. Biología Acuática 14:43.

Carignan, R., D. Planas, and C. Vis. 2000. Planktonic production and respiration in oligotrophic Shield lakes. Limnology and Oceanography 45(1):189-199. https://doi.org/10.4319/1o.2000.45.1.0189.

Cole, J. J., and N. F. Caraco. 1998. Atmospheric exchange of carbon dioxide in a low-wind oligotrophic lake measured by the addition of SF6. Limnology and Oceanography 43(4):647-656. https://doi.org/10.4319/lo.1998.43.4.0647.

Cole, J. J., M. I. Pace, S. R. Carpenter, and J. F. Kitchell. 2000. Persistence of net heteroptrophy in lakes during nutrient addition and food web manipulations. Limnology and Oceanography 45(4):1718- 1730. https://doi.org/10.4319/ lo.2000.45.8.1718.

Cole, J. J., D. L. Bade, D. Bastviken, M. L. Pace, and M. Van De Bogert. 2010. Multiple approaches to estimating air water gas exchange in small lakes. Limnology and Oceanography: Methods 8(6):285-293. https://doi.org/10.4319/ lom.2010.8.285.

Coloso, J. J., J. J. Cole, P. C. Hanson, and M. L. Pace. 2008. Depth-integrated, continuous estimates of metabolism in a 
clear-water lake. Canadian Journal of Fisheries and Aquatic Sciences 65(4):712-722. https://doi.org/10.1139/f08-006.

Coloso, J. J. \}, Cole, J. J. and M. L. Pace. 2011. Difficulty in Discerning Drivers of Lake Ecosystem Metabolism with High-Frequency Data. Ecosystems 14:935. https://doi.org/10.1007/s10021-011-9455-5.

Diovisalvi, N., G. Berasain, F. Unrein, D. Colautti, P. Fermani, M. E. Llames, A. M. Torremorell, L. Lagomarsino, G. Pérez, R. Escaray, J. Bustingorry, M. Ferraro, and H. E. Zagarese. 2010. Chascomús: estructura y funcionamiento de una laguna pampeana turbia. Ecología Austral 20:115-127.

Frenguelli, J. 1950. Rasgos generales de la morfología y la geología de la -Provincia de Buenos Aires. Provincia de Buenos Aires, Ministerio de Obras Publicas de la Provincia de Buenos Aires. Lab. Ensayos Materiales e Investigación Tecnológica (LEMIT) Ser. II, 33:1-72.

González Sagrario, M. A., S. Musazzi, F. Elizalde Córdoba, M. Mendiolar, and A. Lami. 2020. Inferring the occurrence of regime shifts in a shallow lake during the last 250 years based on multiple indicators. Ecological Indicators 117: 106536. https://doi.org/10.1016/j.ecolind.2020.106536.

Hagerthey, S. E., J. J. Cole, and D. Kilbane. 2010. Aquatic metabolism in the Everglades: Dominance of water column heterotrophy. Limnology and Oceanography 55(2):653-666.

https://doi.org/10.4319/lo.2010.55.2.0653.

Herrera, E. C., and K. Nadaoka. 2021. Temporal dynamics and drivers of lake ecosystem metabolism using high resolution observations in a shallow, tropical, eutrophic lake (Laguna Lake, Philippines). Journal of Great Lakes Research 47(4):997-1020. https://doi.org/10.1016/j.jglr.2021.03.017.

Hoellein, T. J., D. A. Bruesewitz, and D. C. Richardson. 2013. Revisiting Odum (1956): A synthesis of aquatic ecosystem metabolism. Limnology and Oceanography, 58(6):2089-2100. https://doi.org/10.4319/lo.2013.58.6.2089.

Izaguirre, I., and A. Vinocur. 1994a. Typology of shallow lakes of the Salado River basin (Argentina), based on phytoplankton communities. Hydrobiologia 277:49-62. https://doi.org/10.1007/BF00023985.

Izaguirre, I., and A. Vinocur. 1994b. Algal assemblages from shallow lakes of the Salado River Basin (Argentina). Hydrobiologia 289(1):57-64. https://doi.org/10.1007/BF00007408.

Klotz, R. L. 2013. Factors driving the metabolism of two north temperate ponds. Hydrobiologia 711(1):9-17. https: //doi.org/10.1007/s10750-013-1450-8.

Laas, A., P. Nõges, T. Kõiv, and T. Nõges. 2012. High-frequency metabolism study in a large and shallow temperate lake reveals seasonal switching between net autotrophy and net heterotrophy. Hydrobiologia 694(1):57-74. https: //doi.org/10.1007/s10750-012-1131-z.

Mariñelarena, A. 1990. Microorganismos indicadores de contaminación en la laguna de Lobos, Pcia. de Bs. As. Revista de la Asociación de Ciencias Naturales del Litoral 21(2):167-173.

Mariñelarena, A. J., and V. H. Conzonno. 1997. Chemical characteristics and trophic status of Lobos pond (Bs. As., Argentina). Natura Neotropicalis 28(1):7-13. https://doi.org/10.14409/natura.v1i28.3692.

Melack, J. M. 2009. Diel variability and community metabolism in African soda lakes. Natural Resources and Environmental Issues 15(1):28.

Odum, H. T. 1956. Primary production in flowing waters. Limnology and Oceanography 1(2):102-117. https://doi.org/ 10.4319/lo.1956.1.2.0102

O'Farrell, I., I. Izaguirre, G. Chaparro, F. Unrein, R. Sinistro, H. Pizarro, P. Rodríguez, P. De Tezanos Pinto, R. Lombardo, and G. Tell. 2011. Water level as the main driver of the alternation between a free-floating plant and a phytoplankton dominated state: A long-term study in a floodplain lake. Aquatic Sciences 73(2):275-287. https://doi.org/10.1007/ s00027-010-0175-2.

Pace, M. L., and Y.T. Prairie. 2005. Respiration in lakes. Chapter 7 in PJ. le B. Williams and P A. del Giorgio(eds.). Respiration in Aquatic Ecosystems. Oxford University Press. https://doi.org/10.1093/acprof:oso/9780198527084.003.0007.

Pozzobon, M. V. 2009. Descripción ambiental de la laguna de Lobos, Pcia de Bs. As. Una propuesta para su monitoreo. Biología Acuática 26:185-195.

Pozzobon, M. V., and G. A. Cabrera. 2013. Abundancia de coliformes en la laguna de Lobos (Buenos Aires) y su relación con el impacto turístico, Instituto Superior de Formación Docente y Técnica Nㅜㄴ, Lobos, Bs. As. VII Congreso de Ecología y Manejo de Ecosistemas Acuáticos Pampeanos. Río Cuarto, Argentina.

Quirós, R., A. M. Rennella, M. A. Boveri, J. J. Rosso, and A. Sosnovsky. 2002. Factores que afectan la estructura y el funcionamiento de las lagunas pampeanas. Ecología Austral 12(2):175-185.

Rubbo, M. J., J. J. Cole, and J. M. Kiesecker 2006. Terrestrial Subsidies of Organic Carbon Support Net Ecosystem Production in Temporary Forest Ponds: Evidence from an Ecosystem Experiment. Ecosystems 9:1170-1176. https: //doi.org/10.1007/s10021-005-0009-6.

Scharfenberger, U., E. Jeppesen, M. Beklioðlu, M. Søndergaard, D. G. Angeler, A. I. Çakýroðlu, S. Drakare, J. Hejzlar, A. Mahdy, E. Papastergiadou, M. Šorf, K. Stefanidis, A. Tuvikene, P. Zingel, and R. Adrian. 2019. Effects of trophic status, water level, and temperature on shallow lake metabolism and metabolic balance: A standardized pan-European mesocosm experiment. Limnology and Oceanography 64(2):1-16. https://doi.org/10.1002/lno.11064.

Staher, P. A., D. Bade, M. C. Van De Bogert, G. R. Koch, C. Williamson, P. Hanson, J. J. Cole, and T. Kratz. 2010. Lake metabolism and the diel oxygen technique: state of the science. Limnology and Oceanography. Methods 8:628-644. https://doi.org/10.4319/lom.2010.8.0628.

Staehr, P. A., L. Baastrup-Spohr, K. Sand-Jensen, et al. 2012. Lake metabolism scales with lake morphometry and catchment conditions. Aquat Sci 74:155-169. https://doi.org/10.1007/s00027-011-0207-6.

Torremorell, A., M., E. Llames, G. Pérez, R. Escaray, J. Bustingorry, and H. Zagarese. 2009. Annual patterns of 
phytoplankton density and primary production in a large, shallow lake: the central role of light. Freshwater Biology 54:437-449. https://doi.org/10.1111/j.1365-2427.2008.02119.x.

Torremorell, A., M. C. Diéguez, C. Queimaliños, I. Izaguirre, and H. E. Zagarese. 2018. Phytoplankton limitation in Patagonian and Pampean shallow lakes: effect of phosphorus and light. Hydrobiologia 816:91-105. https://doi.org/ 10.1007/s10750-017-3181-8.

Van De Bogert, M. C., S. R. Carpenter, J. J. Cole, and M. L. Pace. 2007. Assessing pelagic and benthic metabolism using free water measurements. Limnology and Oceanography: Methods 5(5):145-155. https://doi.org/10.4319/lom.2007.5.145.

Wanninkhof, R. 1992. Relationship between wind speed and gas exchange over the ocean. Journal of Geophysical Research: Oceans 97(C5):7373-7382. https://doi.org/10.1029/92JC00188.

Zbikowski, J.,T. Simcic, F. Pajk, M. Poznanska-Kakareko, T. Kakareko, and J. Kobak. 2019. Respiration rates in shallow lakes of different types: contribution of benthic microorganisms, macrophytes, plankton and macrozoobenthos. Hydrobiologia 828:117-136. https://doi.org/10.1007/s10750-018-3807-5. 